\title{
Feasibility RCT protocol evaluating a powered-wheelchair training program for older adults
}

(c) CAOT 2019

Article reuse guidelines:

sagepub.com/journals-permissions

www.cjotrce.com

@SAGE

\section{Faisabilité du protocole d'un essai randomisé visant à évaluer un programme d'entraînement au fauteuil roulant motorisé pour les aînés}

\author{
Emma M. Smith, William C. Miller, W. Ben Mortenson, \\ and Alex Mihailidis
}

Key words: Aged; Errorless learning; Feasibility studies; Shared control; Wheelchairs.

Mots clés : Âgé; Apprentissage sans erreur; Contrôle partagé; Études de faisabilité; Fauteuils roulants.

\begin{abstract}
Background. Powered-wheelchair use improves participation for people with mobility limitations; however, many individuals do not receive powered-wheelchair skills training that meets their learning needs. Purpose. The aim of this work is to evaluate the feasibility of a powered-wheelchair training program for older adults with cognitive impairment, using errorless learning strategies facilitated by shared control technology. Method. A feasibility $2 \times 2$ factorial randomized controlled trial will recruit 32 older adults in residential care with mild to moderate cognitive impairment who are new powered-wheelchair use. The intervention consists of six or 12 training sessions, facilitated by shared control technology, using errorless learning techniques. Control participants will receive six or 12 training sessions using trial-and-error methods. Feasibility and clinical outcomes data (primary outcome: powered-wheelchair skills) will be collected. Implications. Errorless learning facilitated by shared control technology may be an alternative to meet the powered-wheelchair learning needs of older adults with cognitive impairments.
\end{abstract}

\begin{abstract}
Abrégé
Description. Bien que l'usage d'un fauteuil roulant motorisé permette d'améliorer la participation des personnes ayant des problèmes de mobilité, de nombreux individus ne bénéficient pas d'un programme d'entraînement au fauteuil roulant motorisé qui correspond à leurs besoins en matière d'apprentissage. But. Évaluer la faisabilité d'un programme d'entraînement au fauteuil roulant motorisé pour des aînés ayant des troubles cognitifs basé sur des stratégies d'apprentissage sans erreur facilitées par la technologie d'assistance par contrôle partagé. Méthodologie. Un essai randomisé de faisabilité basé sur un plan factoriel $2 \times 2$ permettra de recruter 32 aînés ayant des troubles cognitifs de léger à modéré qui vivent dans des établissements de soins et qui sont de nouveaux utilisateurs de fauteuils roulants motorisés. L'intervention comprend soit six ou I 2 séances d'entraînement facilitées par la technologie d'assistance par contrôle partagé et basées sur des techniques d'apprentissage sans erreur. Les participants du groupe témoin recevront six ou 12 séances d'entraînement à l'aide de méthodes d'essai et erreur. Des données seront recueillies sur la faisabilité et les résultats cliniques (principaux résultats: habiletés pour utiliser un fauteuil roulant motorisé). Conséquences. L'apprentissage sans erreur facilité par la technologie d'assistance par contrôle partagé peut être une solution de rechange pour répondre aux besoins des aînés ayant des troubles cognitifs qui doivent apprendre à utiliser un fauteuil roulant motorisé.
\end{abstract}

Trial Registration. Clinicaltrials.gov: NCT02982252, December 5, 2016

Funding: AGEWELL NCE and the Promobilia Foundation are providing support for research-related costs and do not play a role in conducting or analyzing the results of this research. ES is supported by a PhD Fellowship from the Canadian Institutes of Health Research and was previously supported by a PhD Scholarship from the Alzheimer Society of Canada. Emma Smith also received a COTF Doctoral scholarship.

Corresponding author: Bill Miller, Department of Occupational Science and Occupational Therapy, University of British Columbia, 22II Wesbrook Mall, Vancouver, BC, V6T2B5, Canada. Telephone: 604-7I4-4107. E-mail: billm@mail.ubc.ca 
$\mathrm{P}$ owered wheelchair (PWC) use improves participation outcomes and quality of life in individuals with mobility impairment through increased independence, social engagement, and mobility and reduced reliance on caregivers (Brandt, Iwarsson, \& Ståhle, 2004; Fomiatti, Richmond, Moir, \& Millsteed, 2013; Lofqvist, Pettersson, Iwarsson, \& Brandt, 2012; Mortenson, Miller, Backman, \& Oliffe, 2011; Pettersson, Törnquist, \& Ahlström, 2006; Salminen, Brandt, Samuelsson, Töytäri, \& Malmivaara, 2009). However, the clinical decision to provide a client with a PWC is complex; it is dependent on an individual's skills and abilities, including cognitive and perceptual abilities; diagnosis and prognosis; and environments of use (Karmarkar et al., 2012; Mortenson, Clarke, \& Best, 2013). As a result, despite the potential benefits of PWC use, many individuals who would benefit do not get access, particularly if they are unable to demonstrate the required skills: capacity to safely negotiate the environment, avoid obstacles (including people), and recognize when assistance is needed (Canning \& Sanchez, 2004).

PWC skills training can be provided to address some of the challenges new users may face and to mitigate the potential risk to the user or others in his or her environment (Kirby, Coughlan, \& Christie, 1995; Mountain et al., 2010). In a study investigating residential-care PWC guidelines, a majority of respondents felt more in-depth training was required, particularly when there are limitations to cognition, movement, or vision (Mortenson et al., 2006). This training ideally considers both the capacities of the learner and the characteristics of the environment of use (Field, 1999; Greer, Brasure, \& Wilt, 2012; Mortenson et al., 2013). However, limitations, including a lack of effective training protocols, available clinical time for training, and concerns about safety in the training process, often result in inadequate training provided to the individuals who need more in-depth or tailored learning opportunities.

Specifically, individuals with cognitive impairments are often denied access to training, or are given training that does not meet their learning needs, limiting their ability to obtain or maintain use of a PWC (Mortenson et al., 2013). Clinicians may be hesitant to engage in PWC training, particularly with learners with cognitive impairments, citing concerns they will not be able to effectively respond to safety issues in the training process. Furthermore, meeting the specific learning needs of cognitively impaired learners is necessary for success. For those with intact cognition, trial-and-error learning can be used, as shortterm working memory allows for recall of the error and correction in subsequent trials. With age-related cognitive decline, shortterm and working memories associated with learning are typically affected (Backman, 1992). Difficulty with verbal recall was associated with challenges remembering operational instructions for the device and the actions that led to errors in a previous trial (Cullen, O'Neill, \& Evans, 2008).

Individuals with cognitive impairment are more likely to learn through errorless training strategies, which do not rely on explicit memory processes for recalling errors (Akhtar, Moulin, \& Bowie, 2006; Baddeley \& Wilson, 1994). Errorless training techniques, including modelling and demonstration, cued learning without and with fading, and spaced retrieval, rely on implicit memory processes that remain relatively intact in the presence of memory loss and other cognitive decline (Davis, 2005; De Vreese \& Neri, 2001). These techniques have been demonstrated to be effective in teaching (or reteaching) procedural skills to individuals with cognitive impairment, including development of morning routines for chronic diabetes management (Ferland, Larente, Rowland, \& Davidson, 2013), activities of daily living and instrumental activities of daily living (Dechamps et al., 2011), and prosthetic limb fitting (Donaghey, McMillan, \& O’Neill, 2010).

Applying errorless learning to powered mobility is a novel approach that has previously not been documented in the literature. To apply these training strategies, clinicians require increased control over the wheelchair to tailor the task to the learner and prevent sources of error. Teleoperation, or shared control, provides a trainer with the opportunity to override the wheelchair user's controls, much like a second steering wheel and brake in a driver-training car. A recent study exploring the clinical utility of shared control for PWC skills assessment and training identified the potential use of the technology for increasing the control provided to the trainer, which would allow alternative approaches to training, similar to those described in the errorless learning literature (Smith, Rismani, Mortenson, \& Miller, in press). Furthermore, clinicians identified the potential for reduced risk in the training environment, which has previously been identified as a training-related barrier (Smith et al., in press). Finally, the reduced risk allows the trainer to increase the amount of training completed in natural environments, which may be more effective for learning for individuals with cognitive impairments and may help to decrease the time required for training (Smith et al., in press).

This study evaluates the feasibility of the Collaborative Power Mobility Innovative Learning OpporTunity (CoPILOT), an errorless approach to powered mobility training, facilitated by shared control technology.

\section{Primary and Secondary Objectives}

The primary objective of this study is to address the feasibility of study methods and procedures for a subsequent large-scale RCT, including process issues of subject recruitment, consent, retention, and perceived benefit; resource issues of treatment adherence and time to complete data collection and intervention; management issues of equipment reliability, subject processing, and protocol administration; and treatment issues of safety, response, and treatment effect (Thabane et al., 2010).

Clinical objectives are secondary to feasibility objectives and are one of the feasibility indicators (treatment response and effect). The primary clinical objective of the study is to evaluate the effects of the intervention (CoPILOT vs. customary-care control) and training dose (six sessions vs. 12 sessions) on PWC skill capacity. The secondary clinical objective of the study is to evaluate the effects of the CoPILOT intervention and training dose on satisfaction and performance of wheelchair-related goals, selfreported PWC skill capacity, PWC skill confidence, and capacity for divided attention in PWC use. 


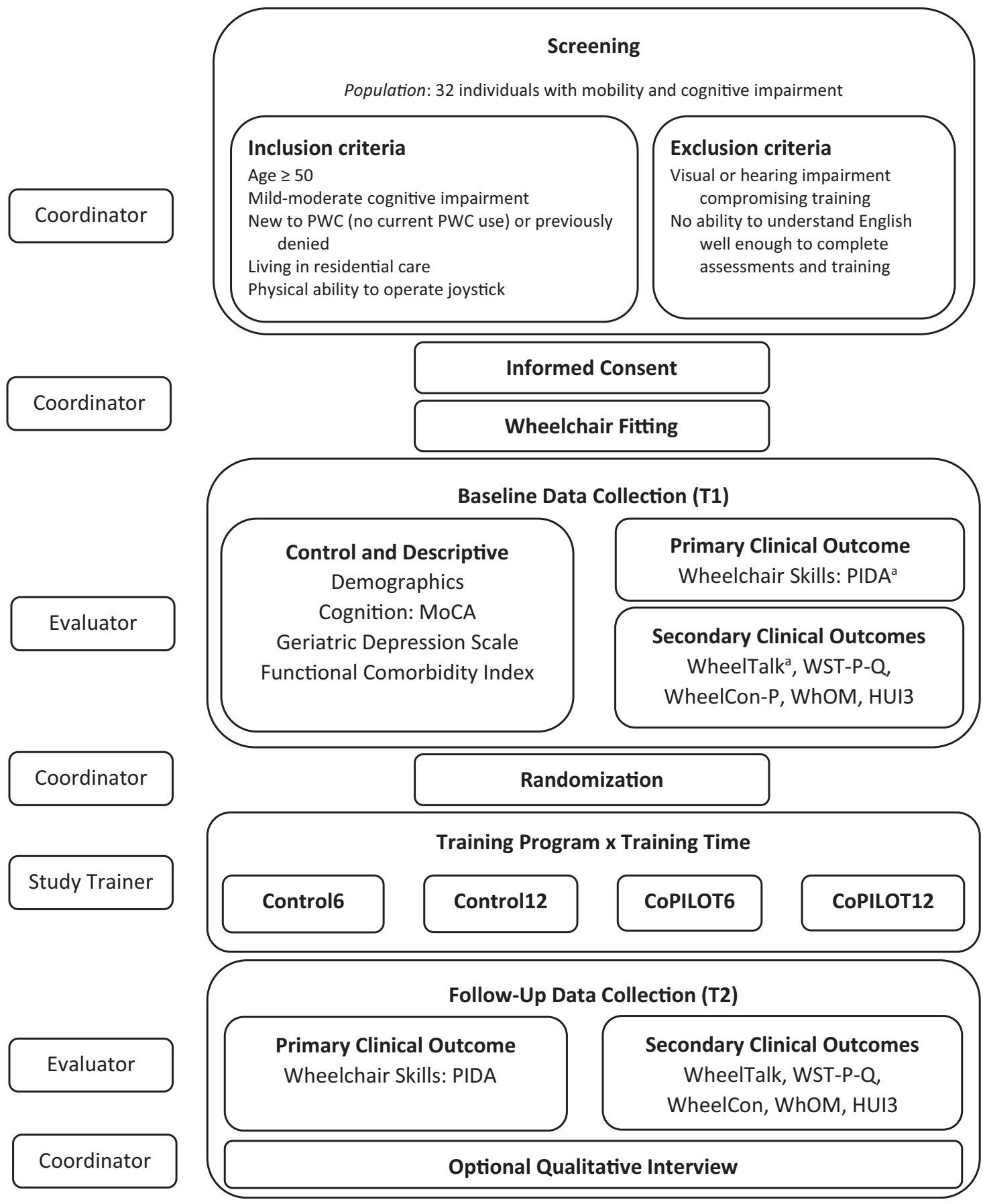

Figure I. CoPILOT study design. PWC = powered wheelchair; MoCA = Montreal Cognitive Assessment; PIDA = Power-Mobility Indoor Driving Assessment; WheelTalk = Wheeling While Talking Test; WhOM = Wheelchair Outcome Measure; WST-P-Q = Wheelchair Skills Test for Powered Wheelchair Users Questionnaire; WheelCon-P = Wheelchair Use Confidence Scale for Powered Wheelchairs; HUI3 = Health Utility Index 3; CoPILOT = Collaborative Power Mobility Innovative Training OpporTunity.

${ }^{a}$ Completed at baseline only for participants with previous PWC experience.

\section{Description of the Trial Design}

A $2 \times 2$ factorial, evaluator-blinded feasibility RCT will evaluate the feasibility of study methods for use in a large-scale RCT. This study will investigate two intervention factors: type of training (CoPILOT vs. customary-care control) and training time (six sessions vs. 12 sessions), their effects on the primary and secondary clinical outcomes, and potential interactions between factors. A visual representation is provided in Figure 1. 


\section{Method}

\section{Description of Participants}

Participants $(N=32)$ will be included if they live in residential care, are $\geq 50$ years old, may benefit from the use of a PWC, can operate a standard PWC joystick, and score between 18 and 26 on the Mini-Mental State Examination (consistent with mild to moderate cognitive impairment; Tombaugh \& McIntyre, 1992). Participants may be new to PWC use or previously denied due to safety concerns. Participants will be excluded if their clinical therapist has identified visual and/or hearing concerns that may compromise training safety or if they are unable to speak, read, or write English well enough to complete study outcome measures.

As the primary objective of this study is to assess the feasibility of study procedures, it may not be appropriate to determine sample size based on calculations from the primary outcome (Billingham, Whitehead, \& Julious, 2013; Hertzog, 2008). A sample size of 32 (eight per group; 16 per factor) was selected to ensure sufficient replication to address feasibility outcomes and ensure precision of means and variance for feasibility outcomes.

\section{Interventions}

The CoPILOT intervention will use a PWC skills training approach that emphasizes errorless, experiential learning facilitated by shared control technology used by the trainer. The intervention will take place in familiar environments in each participant's residential care facility. During training, participants will independently operate the PWC with the trainer offering verbal and visual cues and guidance through the shared control technology to prevent a collision or unsafe event, demonstrate a skill, or promote experiential learning. For example, if a participant is learning to manoeuvre the chair through a hallway and is at risk of hitting the sidewall, the trainer will have the capacity to gently guide the chair away from the wall, demonstrate proper driving techniques, and prevent a collision. Trainers will also have the capacity to modify speed (acceleration and deceleration) and turning direction as necessary or to engage the emergency stop function to prevent an unsafe event.

Skills will be introduced and progressed using training techniques that gradually increase potential error and effort in skill retrieval, which has been shown to be important to learning (Middleton \& Schwartz, 2012; Mimura \& Komatsu, 2007; see Figure 2). An example of skill progression using errorless techniques is provided in Table 1. The CoPILOT Training Manual is available from the authors.

The control (customary care) protocol uses the Wheelchair Skills Program (Kirby et al., 2018). Trainers will provide instructions on PWC skills through verbal or visual instruction, using trial-and-error methods. Skills will progress from basic to advanced, building on success of previously learned skills. Participants will be required to consistently demonstrate safe operation of the device in a quiet environment with nonhuman obstacles prior to proceeding to complex environments with people present. Training will be completed in a wheelchair comparable to that used in the CoPILOT protocol but without the shared control capacity. This will minimize potential differences in the training program that are not attributable to the CoPILOT approach. In circumstances where a trainer feels the participant is at risk to himself or herself or to others, the trainer will verbally ask the participant to stop. If the situation is more urgent, the trainer may remove the participant's hand from the joystick or turn off the chair.

\section{Total Training Time}

This study will assess both the intervention and control protocols at two levels of training time (six vs. 12 sessions) to determine if there is an additional effect of time and to determine feasibility differences between these intervals. As there is no standard dose of training provided in clinical practice, and no published evidence regarding an effective dose of training, these times are consistent with published data regarding training protocols in two Canadian facilities, which found evidence of wheelchair skill acquisition at both six and 12 sessions (Hall, Partnoy, Tenenbaum, \& Dawson, 2005). Participants completing six sessions will complete three sessions per week over 2 weeks; participants completing 12 sessions will complete four sessions per week over 3 weeks. Sessions will last a maximum of $1 \mathrm{hr}$, dependent on the training tolerance of the participant.

\section{Intervention Fidelity}

Trainers will be provided with education regarding the protocols, including the theoretical underpinnings of the CoPILOT and control protocols. All trainers will be provided with an intervention manual (CoPILOT or control) that outlines the protocol in detail and provides suggested training progressions and information about training techniques. All trainers will also have the opportunity to practise skills required for the delivery of the intervention, including technical skills for use of the CoPILOT shared control system (if applicable) and the standard wheelchair. Finally, new CoPILOT trainers will shadow a minimum of two training sessions with an experienced CoPILOT trainer.

Throughout the study, fidelity of the intervention delivery will be monitored through regular audit of staff logs, where trainers will report skills and techniques used in the training process and challenges adhering to the protocol, and through regular team meetings with the investigators and study coordinator. Trainers will exclusively deliver either the CoPILOT or the control intervention to ensure there is no contamination between protocols.

\section{Equipment}

The PWC used for both the CoPILOT and control interventions will be a standard mid-wheel drive PWC, most often used in residential care environments (Sabol \& Haley, 2006), adjusted to the participant's size and featuring powered tilt to promote stability and positioning for posture or pressure management. PWCs outfitted with shared control technology will be used for participants receiving the CoPILOT intervention. 


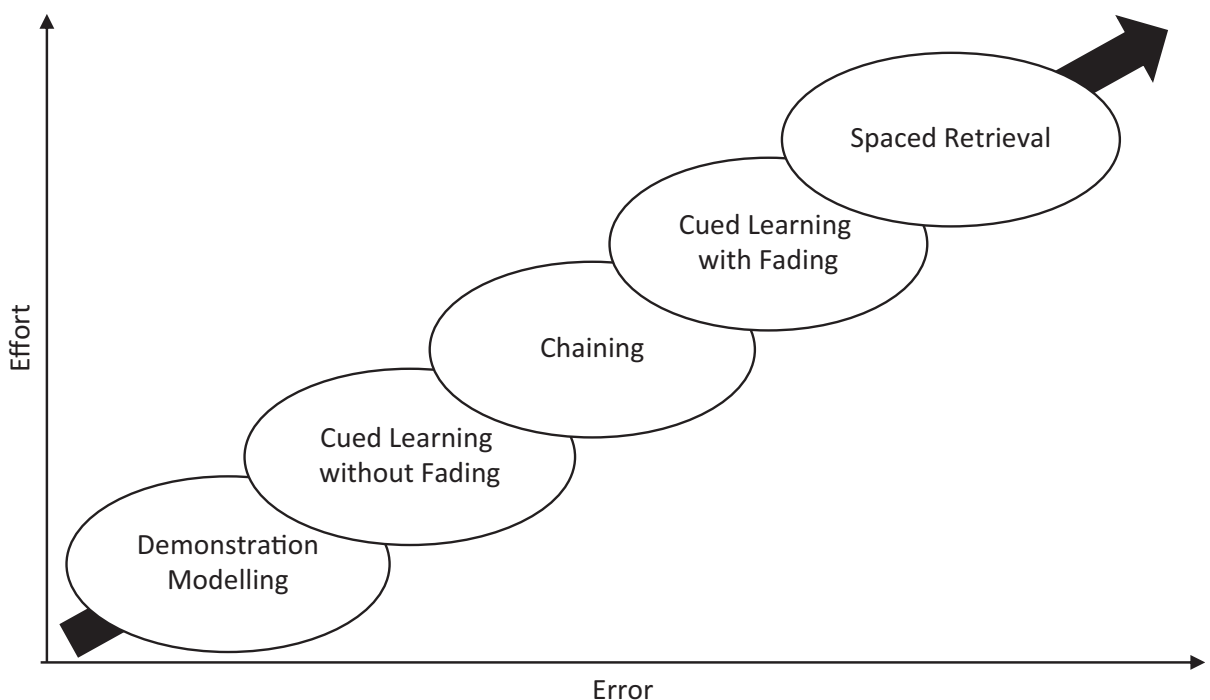

Figure 2. Training-technique skill progression.

Table I

Sample Skill Progression Using an Error-Minimized Approach for the Skill "Navigating an Elevator"

\begin{tabular}{|c|c|c|}
\hline Training technique & $\begin{array}{l}\text { Level of error/ } \\
\text { effort }\end{array}$ & Description \\
\hline \multirow[t]{6}{*}{$\begin{array}{l}\text { Demonstration/ } \\
\text { modelling }\end{array}$} & Low/low & $\begin{array}{l}\text { Using the CoPILOT remote, execute the elevator skill providing verbal and visual cues for each step. } \\
\text { Steps are as follows (key considerations in parentheses): }\end{array}$ \\
\hline & & I) Approach the elevator (orientation in space, speed) \\
\hline & & 2) Call the elevator (distance, spacing) \\
\hline & & 3) Position to enter the elevator (spacing, planning entrance, awareness of others) \\
\hline & & 6) Operate elevator (position, spacing) \\
\hline & & 7) Exit elevator (awareness of others, speed of doors, chair speed, joystick use) \\
\hline \multirow[t]{2}{*}{$\begin{array}{l}\text { Cued learning } \\
\text { without fading }\end{array}$} & Low/low & $\begin{array}{l}\text { Instruct the learner to call, enter, operate, and exit the elevator, providing continuous cues regarding } \\
\text { joystick use, orientation, spacing, awareness of others, and speed as outlined above. }\end{array}$ \\
\hline & & Provide override assistance as necessary to minimize potential risk, including risk of collision. \\
\hline $\begin{array}{l}\text { Cued learning with } \\
\text { fading }\end{array}$ & $\begin{array}{l}\text { Medium/ } \\
\text { medium }\end{array}$ & As above, with fewer cues each subsequent trial. Reduce override assistance where possible. \\
\hline & & $\begin{array}{l}\text { Backward chaining: Using the CoPILOT remote, complete Steps I through 6, and instruct learner to } \\
\text { complete the final skill (exit elevator), helping where necessary. Add skills in reverse order on each } \\
\text { subsequent trial until the learner is completing the entire skill independently. }\end{array}$ \\
\hline & & $\begin{array}{l}\text { Note: You may wish to mix these techniques to have the learner complete those steps he or she is } \\
\text { comfortable with, adding steps in order of difficulty. }\end{array}$ \\
\hline Spaced retrieval & High/high & $\begin{array}{l}\text { During initial training, ensure skill is repeated regularly. As the learner gains proficiency, reduce the } \\
\text { number of times the skill is performed until elevator use is limited to functional needs. }\end{array}$ \\
\hline
\end{tabular}

Note. CoPILOT $=$ Collaborative Power Mobility Innovative Learning OpporTunity.

\section{Outcomes}

Feasibility indicators will be collected for process, resource, management, and treatment outcomes throughout the study administration and at completion. Table 2 outlines criteria for success for each of the feasibility indicators.
To assess treatment outcomes, we will collect a variety of data, including control and descriptive measures and primary, secondary, and tertiary clinical outcomes. Table 3 outlines all measures, including constructs evaluated and time points for data collection. 
Table 2

Feasibility Indicators and Parameters for Success

\begin{tabular}{|c|c|c|}
\hline Feasibility indicator & Measure & Parameter for success \\
\hline \multicolumn{3}{|l|}{ Process } \\
\hline Recruitment rate & Number of subjects recruited & $\begin{array}{l}4 \text { subjects per month: } \\
\text { Total of } 32 \text { over } 8 \text { months }\end{array}$ \\
\hline Consent rate & $\%$ of subjects consenting & $<10 \%$ subject refusal \\
\hline Retention rate & $\%$ of subjects with $\mathrm{T} 2$ data collected & Complete T2 data collection with $>80 \%$ of subjects \\
\hline Perceived benefit & Qualitative interviews with participants and trainers & $\begin{array}{l}\text { Qualitative analysis will inform perceived benefit and } \\
\text { clinical significance }\end{array}$ \\
\hline \multicolumn{3}{|l|}{ Treatment adherence } \\
\hline CoPILOT group & Number of training sessions attended & $\begin{array}{l}>85 \% \text { of subjects attend } 12 \text { sessions ( } 12 \text {-session } \\
\text { protocol) } \\
>85 \% \text { of subjects attend six sessions (six-session } \\
\text { protocol) }\end{array}$ \\
\hline \multicolumn{3}{|l|}{ Data collection } \\
\hline $\begin{array}{l}\text { Subject and evaluator } \\
\text { time }\end{array}$ & Time to complete data collection & $\begin{array}{l}>85 \% \text { of subjects complete } \mathrm{TI} \leq 2 \mathrm{hr} \\
>85 \% \text { of subjects complete } \mathrm{T} 2 \leq 1.5 \mathrm{hr}\end{array}$ \\
\hline Collection of HUI3 data & Time to administer HUI3 pre-/post-treatment score & $\begin{array}{l}\text { Mean administration is }<10 \mathrm{~min} \\
\text { Statistically significant } \mathrm{TI} \text { and } \mathrm{T} 2 \text { change }\end{array}$ \\
\hline \multicolumn{3}{|l|}{ Trainer time } \\
\hline CoPILOT group & Time spent on training intervention & $\begin{array}{l}\text { Mean time spent per subject is }<20 \mathrm{hr} \text { (12-session } \\
\text { protocol) } \\
\text { Mean time spent per subject is }<10 \mathrm{hr} \text { (six-session } \\
\text { protocol) }\end{array}$ \\
\hline Control group & Time spent on training intervention & $\begin{array}{l}\text { Mean time spent per subject is }<20 \mathrm{hr} \text { (12-session } \\
\text { protocol) } \\
\text { Mean time spent per subject is }<10 \mathrm{hr} \text { (six-session } \\
\text { protocol) }\end{array}$ \\
\hline \multicolumn{3}{|l|}{ Treatment } \\
\hline Safety (skills training) & Adverse events during skills training & No major injuries or adverse events reported \\
\hline $\begin{array}{l}\text { Safety (data collection and } \\
\text { assessment) }\end{array}$ & Adverse events during assessment & No major injuries or adverse events reported \\
\hline Treatment response & Two-way ANOVA comparison between groups & A significant difference between groups identified \\
\hline Dose level response & Two-way ANOVA comparison between groups & At least one training dose sufficient for a treatment effect \\
\hline $\begin{array}{l}\text { Treatment effect and } \\
\text { variance }\end{array}$ & $\begin{array}{l}\text { Estimate of effect size and variance for future sample } \\
\text { size/power calculations }\end{array}$ & \\
\hline
\end{tabular}

Note. CoPILOT = Collaborative Power Mobility Innovative Learning OpporTunity; HUI3 = Health Utility Index 3; TI = Time I; T2 = Time 2.

The primary clinical outcome (PWC skill) will be assessed using the Power-Mobility Indoor Driving Assessment (PIDA). The PIDA measures capacity and safety of driving skill in a residential care environment. The PIDA is the only reliable tool available to measure wheelchair skills, which include global skills of speed selection and sharing public spaces, addressing issues of judgement and insight that may be challenged with impaired cognition (Dawson, Chan, \& Kaiserman, 1994).

\section{Assignment of Interventions}

Following enrolment and collection of demographic and baseline data, participants will be randomized to one of four groups (CoPILOT6, CoPILOT12, Control6, Control12) using an online computerized process (www.sealedenvelope.com), which will balance groups using a random permuted blocks design. Block sizes between four and 16 will be used, with allocation concealed prior to randomization. 
Table 3

Outcome Measures

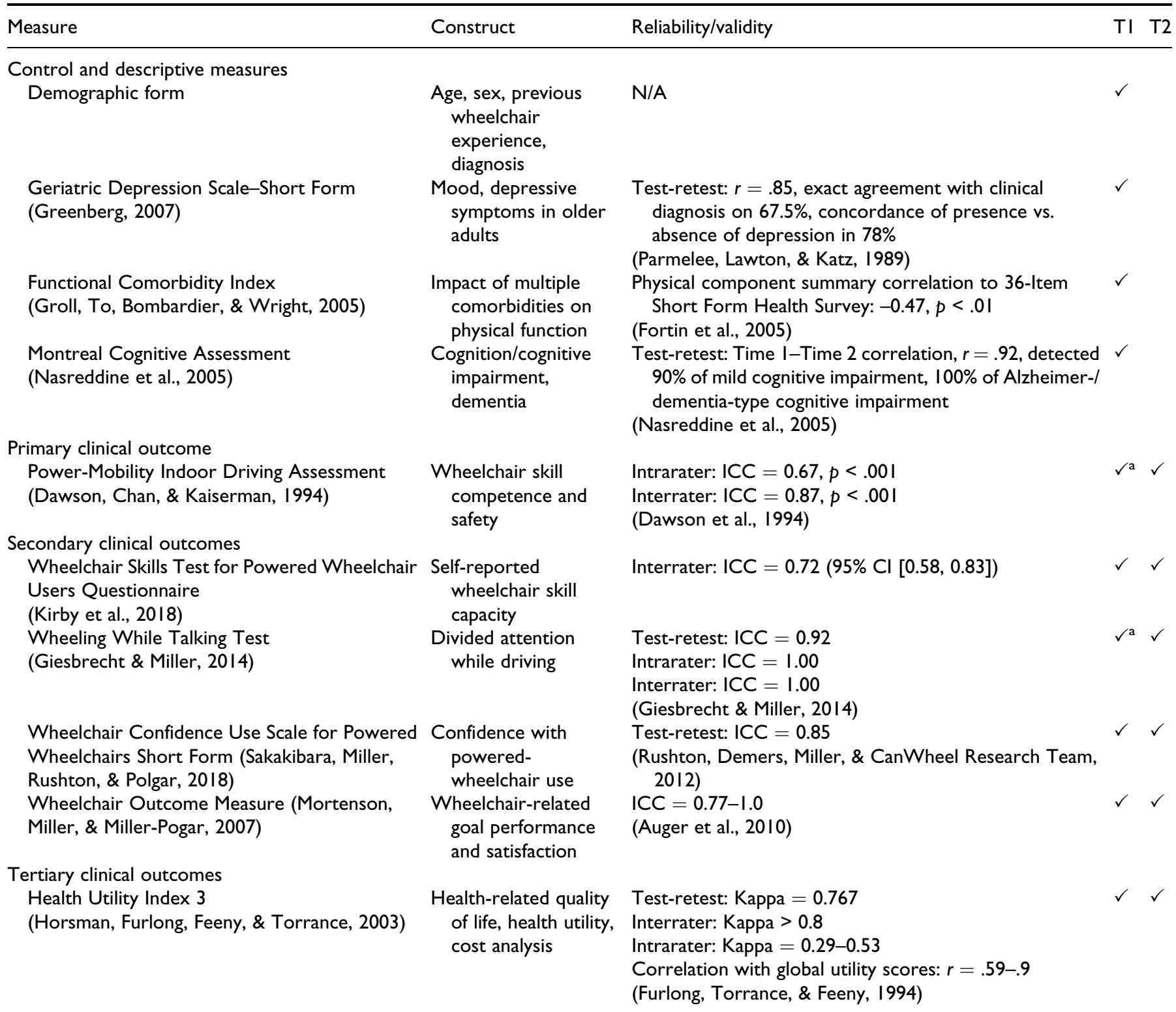

Note. ICC = intraclass correlation; $\mathrm{Cl}=$ confidence interval.

${ }^{a}$ Completed at baseline only for participants with previous powered-wheelchair experience.

\section{Description of Data Collection}

Feasibility data will be collected using staff logs and qualitative interviews with the study trainers, evaluators, and coordinator and during collection of clinical-outcomes data. Training logs will be completed following each training session to collect information on skills practised, safety or equipment concerns, adverse events, and a plan for the subsequent training session. Throughout the study, staff will record challenges with data management and coordination. Semistructured qualitative interviews will provide further data regarding feasibility outcomes and focus on protocol changes to maximize success in a future RCT (see Figure 3 for sample interview questions).
Clinical outcomes will be measured by a trained and blinded evaluator at baseline (pre-randomization; Time 1 [T1]) and following completion of participant training (Time 2 [T2]). At T1, all participants will complete control and descriptive measures, the Wheelchair Use Confidence Scale for Powered Wheelchair Users (WheelCon-P), Wheelchair Outcome Measure (WhOM), and Wheelchair Skills Test for Powered Wheelchair Users Questionnaire (WST-P-Q). If participants indicate previous experience with a $\mathrm{PWC}$ and report capacity to operate the PWC for a variety of basic driving tasks (driving in a straight line and stopping, turning left and right), they will also complete baseline evaluations for the PIDA and 


\section{Participants}

I. Please tell me about your experience learning to drive the powered wheelchair.

2. What things did the trainer do that made it easier for you to learn how to drive the wheelchair?

3. What things could the trainer have done to help you learn the skills more easily?

4. Please tell me about how safe you felt while learning to drive the powered wheelchair.

Trainers

I. Please describe how you used the CoPILOT system when training throughout the study.

2. What were the benefits of the CoPILOT system in your training?

3. What difficulties did you encounter using the CoPILOT system?

4. Please tell me about your experience using the study protocol.

5. If you were to complete this study again what changes would you make to the study protocol, and why?

Evaluators

I. Please describe any difficulties you had administering the evaluation.

2. Please describe your experience conducting the evaluation with the electronic or paper-based forms.

3. What was your experience of booking and completing the evaluations with participants.

Coordinator

I. Please describe your experience managing the wheelchairs and CoPILOT systems required for the study.

2. What was your experience with participation recruitment and retention?

3. If you were to complete this study again, what changes would you make to the study protocol, and why?

Figure 3. Sample interview questions.

Wheeling While Talking Test (WheelTalk). Participants who have never driven a PWC before will not complete these measures at T1 due to safety concerns. Following training (T2), all participants will be asked to complete all clinical outcome measures.

\section{Data Management}

Data will be collected using electronic and paper-based data forms. All data will be deidentified, entered into a secure database, and checked by a second person for accuracy. Hard copies of data will be kept in a locked filing cabinet in the primary investigator's research lab.

\section{Data Analysis}

Feasibility outcomes reported in statistical analysis will include mean administration time for testing of all clinical outcome measures. The remaining feasibility outcomes will be coded as either successful or requiring revision for a future clinical trial. Standards for success have been set a priori and indicate a specific aspect of the protocol can be used in the future with few or no changes (see Table 2).

Clinical data will include control and descriptive variables (i.e., age, sex, presence of depression) and clinical-outcome measures. Measures of central tendency with standard deviation will be produced for all continuous demographic and baseline data, as will clinical-outcome measures. Frequency and proportion will be reported for baseline categorical variables.

Post-treatment PIDA scores will be compared for main effects and potential interactions within and between factors (intervention and training time) using two-way analysis of variance (ANOVA). Effect size estimates for PIDA scores will be calculated to be used in a future RCT. Descriptive data and scores from control measures will be included in multivariate analysis to control for any confounding influences on the primary outcome. Significance testing $(p)$ and marginal means with $95 \%$ confidence intervals will be estimated. Effect size (partial $\eta^{2}$ ) will be calculated as a ratio of the effect and total sums of squares, with a $95 \%$ confidence interval. Missing data will be handled using multiple imputation.

Secondary analysis. A linear mixed model will be used to compare post-treatment scores for wheelchair-related goal satisfaction and performance (WhOM), self-reported wheelchair skills (WST-P-Q) scores, wheelchair skill confidence (WheelCon-P), and divided attention (WheelTalk) scores for both intervention and training time factors and any potential interactions. Significance testing $(p)$ and $95 \%$ confidence intervals will be estimated.

Post-treatment qualitative analysis. Analysis of qualitative data will be conducted by two investigators, using a directed content analysis approach (Hsieh \& Shannon, 2005), with analyses guided by feasibility parameters described in Table 2 .

\section{Safety and Monitoring}

Safety will be promoted using a combination of wheelchairrelated safety features and trainer judgment. Wheelchairrelated features will include set maximal speed, appropriate seating and safety/positioning belts, and an emergency stop protocol. The trainer will be responsible for ensuring safety throughout the training period for the participant and others in the environment. Adverse events will be reported to the Clinical Research Ethics Board and followed up in the study protocol. Safety, including reports of adverse events (e.g., collisions causing injury to the participant or others, falls during training), is one of the feasibility indicators.

\section{Ethics}

Informed consent. This study may include individuals who do not have capacity to provide informed consent; therefore we will include proxy decision makers where necessary. Legal consent will be signed by the participant and/or a substitute decision maker, and an ongoing process of consent monitoring will be used (Hubbard, Downs, \& Tester, 2003). As in standard clinical practice, the trainer will provide a reminder to the participant at the outset of each training session about the 
process of the study and seek verbal consent to proceed. Process consent will be documented in the training log.

Research ethics approval. This study protocol was approved by the Clinical Research Ethics Board at the University of British Columbia, Vancouver Coastal Health Research Institute, and Providence Health Care.

Confidentiality. All identifying materials will be removed from the data files (both hard copy and electronic), with study numbers used to identify participants. This unique subject number will not be derived from personal identifiers.

\section{Discussion}

\section{Potential Impact and Significance of the Study}

This study will contribute to the justification for a larger RCT to assess the effectiveness of the CoPILOT intervention for individuals with cognitive impairment in residential care. An aging population, with an associated increase in cognitive impairments, requires training approaches that are tailored to their needs. The use of novel technology to facilitate these approaches will bridge the gap between standard training and learning needs of these individuals. Demonstrating the effectiveness of an errorless training approach for this population in a future RCT will also inform best practice for PWC skills training. As many individuals in residential care are wheelchair users (Shields, 2004), this study has the potential to impact a large number individuals who may have previously been deemed unsuitable for PWC use. As a result, this intervention could increase the number of individuals eligible for PWC use and provide secondary benefits in terms of increased independence, participation, and quality of life.

The inclusion of time for training as an intervention variable will provide preliminary evidence about the effectiveness of training dose for PWC training. As clinicians identify time as a barrier to providing wheelchair skills training, this could inform clinical practice and contribute to the development of best-practice standards.

Shared control has been identified as having potential to increase safety in the training process (Smith et al., in press). Safety has been identified as a key area of concern for clinicians when conducting training and may contribute to the decision not to proceed with training for individuals with cognitive impairments (Smith, Kenyon, Field, \& Miller, 2017). While this study specifically focuses on older adults with cognitive impairments, demonstrating feasibility for this intervention in a clinical trial may support future investigations of a technologysupported errorless learning approach for use with additional populations who experience cognitive impairment, including individuals with developmental disability or brain injury.

This study relies on technology not yet available on the market and not previously validated for use in training. A recent study evaluating the potential clinical utility of shared control identified several potential benefits and drawbacks to the use of shared control for training (Smith et al., in press). While participants were generally positive about the potential application of shared control, some concern was raised regarding the need for appropriate feedback to the learner, to mitigate the potential he or she might not understand the behaviour of the wheelchair when it is being overridden by the trainer (Smith et al., in press). Concerns were also raised regarding the need for ongoing trainer practice to maintain competence with the device (Smith et al., in press). Success in this trial will provide an opportunity to further assess these concerns in a larger trial, focused on the efficacy of the technology for PWC skills training.

\section{Limitations}

This study addresses the feasibility of study procedures for a future RCT and does not address skill retention over time. It is also unable to address the potential future needs of those who may experience further decline in cognitive ability and the risk associated with this decline. These limitations should be addressed in a future large-scale RCT.

As customary care differs by institution, it has been standardized in this study to ensure it is comparable in duration to the CoPILOT treatment, minimizing differences in trainer attention. Results from the control intervention may not be comparable to that received in all residential care facilities locally or in other jurisdictions.

\section{Conclusion}

This study is the first to evaluate the use of error-minimized training techniques and dose required for PWC skills training. There is currently minimal research evidence for PWC skills training in the literature. Success in this study will provide justification for proceeding to a larger, multisite RCT to evaluate the effectiveness of the training program. Furthermore, this study will provide effect size estimates for both the primary outcome (PIDA) and for training dose, which will help to assess sample size for future research in PWC skills training. The development and evaluation of an evidence-based training program for PWC skills training will inform clinical practice and contribute to future best-practice standards.

\section{Key Messages}

- Powered-wheelchair skills training based in trial-and-error methods may not provide the best learning opportunities for individuals with cognitive and memory impairments.

- Shared control technology may provide opportunities for safe and effective powered-wheelchair skills training.

- There is minimal research evidence supporting poweredwheelchair skills training. Evidence-based programs for powered wheelchair skills training are needed. 


\section{References}

Akhtar, S., Moulin, C. J. A., \& Bowie, P. C. W. (2006). Are people with mild cognitive impairment aware of the benefits of errorless learning? Neuropsychological Rehabilitation, 16, 329-346. doi:10. 1080/09602010500176674

Auger, C., Demers, L., Gélinas, I., Routhier, F., Mortenson, W. B., \& Miller, W. C. (2010). Reliability and validity of telephone administration of the Wheelchair Outcome Measure for middleaged and older users of power mobility devices. Journal of Rehabilitation Medicine, 42, 574-581. doi:10.2340/165019770557

Backman, L. (1992). Memory training and memory improvement in Alzheimer's disease: Rules and exceptions. Acta Neurological Scandinavia, 139, 84-89.

Baddeley, A., \& Wilson, B. A. (1994). When implicit learning fails: Amnesia and the problem of error elimination. Neuropsychologia, 32(1), 53-68.

Billingham, S. A. M., Whitehead, A. L., \& Julious, S. A. (2013). An audit of sample sizes for pilot and feasibility trials being undertaken in the United Kingdom registered in the United Kingdom Clinical Research Network database. BMC Medical Research Methodology, 13, Article 104. doi:10.1186/14712288-13-104

Brandt, Å., Iwarsson, S., \& Ståhle, A. (2004). Older people's use of powered wheelchairs for activity and participation. Journal of Rehabilitation Medicine, 36, 70-77. doi:10.1080/16501970310017432

Canning, B., \& Sanchez, G. (2004). Innovations in practice: Considering powered mobility for individuals with stroke. Topics in Stroke Rehabilitation, 11(2), 84-88.

Cullen, B., O’Neill, B., \& Evans, J. J. (2008). Neuropsychological predictors of powered wheelchair use: A prospective follow-up study. Clinical Rehabilitation, 22, 836-46. doi:10.1177/ 0269215508091873

Davis, L. A. (2005). Educating individuals with dementia. Topics in Geriatric Rehabilitation, 21, 304-314. doi:10.1097/00013614200510000-00007

Dawson, D., Chan, R., \& Kaiserman, E. (1994). Development of the Power-Mobility Indoor Driving Assessment for residents of longterm care facilities: A preliminary report. Canadian Journal of Occupational Therapy, 61, 269-276. doi:10.1177/000841749406 100507

Dechamps, A., Fasotti, L., Jungheim, J., Leone, E., Dood, E., Allioux, A., ... Kessels, R. P. C. (2011). Effects of different learning methods for instrumental activities of daily living in patients with Alzheimer's dementia: A pilot study. American Journal of Alzheimer's Disease and Other Dementias, 26, 273-281. doi:10.1177/ 1533317511404394

Donaghey, C. L., McMillan, T. M., \& O’Neill, B. (2010). Errorless learning is superior to trial and error when learning a practical skill in rehabilitation: A randomized controlled trial. Clinical Rehabilitation, 24, 195-201. doi:10.1177/0269215509353270

Ferland, M. B., Larente, J., Rowland, J., \& Davidson, P. S. R. (2013). Errorless (re)learning of daily living routines by a woman with impaired memory and initiation: Transferrable to a new home? Brain Injury, 27, 1461-1469. doi:10.3109/02699052.2013.823661
Field, D. (1999). Powered mobility: A literature review illustrating the importance of a multifaceted approach. Assistive Technology, 11(1), 20-33. doi:10.1080/10400435.1999.10131982

Fomiatti, R., Richmond, J., Moir, L., \& Millsteed, J. (2013). A systematic review of the impact of powered mobility devices on older adults' activity engagement. Physical \& Occupational Therapy in Geriatrics, 31, 297-309. doi:10.3109/02703181.2013. 846451

Fortin, M., Hudon, C., Dubois, M.-F., Almirall, J., Lapointe, L., \& Soubhi, H. (2005). Comparative assessment of three different indices of multimorbidity for studies on health-related quality of life. Health and Quality of Life Outcomes, 3, Article 74. doi:10. 1186/1477-7525-3-74

Furlong, W., Torrance, G. W., \& Feeny, D. (1994). Properties of Health Utilities Index: Preliminary evidence. Quality of Life Newsletter, 13/14, 3, 4, 10.

Giesbrecht, E. M., \& Miller, W. C. (2013, June). Development of the wheeling while talking test. Paper presentation at the Rehabilitation Engineering and Assistive Technology Society of North America Conference, Bellevue, WA.

Greenberg, S. A. (2007). The Geriatric Depression Scale: Short form. American Journal of Nursing, 107, 60-69. doi:10.1097/01.NAJ. 0000292204.52313.f3

Greer, N., Brasure, M., \& Wilt, T. (2012). Wheeled mobility (wheelchair) service delivery: Scope of the evidence. Annals of Internal Medicine, 156(2), 141-146.

Groll, D. L., To, T., Bombardier, C., \& Wright, J. G. (2005). The development of a comorbidity index with physical function as the outcome. Journal of Clinical Epidemiology, 58, 595-602. doi:10. 1016/j.jclinepi.2004.10.018

Hall, K., Partnoy, J., Tenenbaum, S., \& Dawson, D. R. (2005). Power mobility driving training for seniors: A pilot study. Assistive Technology, 17(1), 47-56. doi:10.1080/10400435.2005.10132095

Hertzog, M. A. (2008). Considerations in determining sample size for pilot studies. Research in Nursing \& Health, 31(2), 180-191. doi: 10.1002/nur.20247

Horsman, J., Furlong, W., Feeny, D., \& Torrance, G. (2003). The Health Utilities Index (HUI): Concepts, measurement properties and applications. Health and Quality of Life Outcomes, 1, 54. doi:10.1186/1477-7525-1-54

Hsieh, H.-F., \& Shannon, S. E. (2005). Three approaches to qualitative content analysis. Qualitative Health Research, 15, 1277-1288. doi: $10.1177 / 1049732305276687$

Hubbard, G., Downs, M. G., \& Tester, S. (2003). Including older people with dementia in research: Challenges and strategies. Aging \& Mental Health, 7(5), 351-362. doi:10.1080/1360786031000150685

Karmarkar, A. M., Dicianno, B. E., Graham, J. E., Cooper, R., Kelleher, A., \& Cooper, R. A. (2012). Factors associated with provision of wheelchairs in older adults. Assistive Technology, 24, 155-167. doi:10.1080/10400435.2012.659795

Kirby, R. L., Coughlan, S. G., \& Christie, M. (1995). Could changes in the wheelchair delivery system improve safety? Canadian Medical Association Journal, 153, 1585-1591.

Kirby, R. L., Rushton, P., Smith, C., Routhier, F., Best, K., Cowan, R., . . . Worobey, L. (2018). Wheelchair Skills Program manual. Retrieved from http://www.wheelchairskillsprogram.ca/eng/manual.php 
Lofqvist, C., Pettersson, C., Iwarsson, S., \& Brandt, A. (2012). Mobility and mobility-related participation outcomes of powered wheelchair and scooter interventions after 4-months and 1-year use. Disability \& Rehabilitation: Assistive Technology, 7, 211-218. doi:10.3109/17483107.2011.619244

Middleton, E. L., \& Schwartz, M. F. (2012). Errorless learning in cognitive rehabilitation: A critical review. Neuropsychological Rehabilitation, 22(2), 1-24. doi:10.1080/09602011.2011.639619

Mimura, M., \& Komatsu, S. (2007). Cognitive rehabilitation and cognitive training for mild dementia. Psychogeriatrics, 7(3), 137-143. doi:10.1111/j.1479-8301.2007.00212.x

Mortenson, W., Clarke, L., \& Best, K. (2013). Prescribers' experiences with powered mobility prescription among older adults. American Journal of Occupational Therapy, 67, 100-107.

Mortenson, W., Miller, W. C., Backman, C. L., \& Oliffe, J. L. (2011). Predictors of mobility among wheelchair using residents in longterm care. Archives of Physical Medicine \& Rehabilitation, 92, 1587-1593. doi:10.1016/j.apmr.2011.03.032

Mortenson, W. B., Miller, W. C., Boily, J., Steele, B., Crawford, E. M., \& Desharnais, G. (2006). Overarching principles and salient findings for inclusion in guidelines for power mobility use within residential care facilities. Journal of Rehabilitation Research \& Development, 43(2), 199-208.

Mortenson, W. B., Miller, W. C., \& Miller-Polgar, J. (2007). Measuring wheelchair intervention outcomes: Development of the wheelchair outcome measure. Disability and Rehabilitation: Assistive Technology, 2, 275-285. doi:10.1080/17483100701475863

Mountain, A. D., Kirby, R. L., Eskes, G. A., Smith, C., Duncan, H., MacLeod, D. A., \& Thompson, K. (2010). Ability of people with stroke to learn powered wheelchair skills: A pilot study. Archives of Physical Medicine and Rehabilitation, 91, 596-601. doi:10. 1016/j.apmr.2009.12.011

Nasreddine, Z. S., Phillips, N. A., Bédirian, V., Charbonneau, S., Whitehead, V., Collin, I., ... Chertkow, H. (2005). The Montreal Cognitive Assessment, MoCA: A brief screening tool for mild cognitive impairment. Journal of the American Geriatrics Society, 53, 695-699. doi:10.1111/j.1532-5415.2005.53221.x

Parmelee, P. A., Lawton, M. P., \& Katz, I. R. (1989). Psychometric properties of the Geriatric Depression Scale among the institutionalized aged. Psychological Assessment, 1, 331-338. doi:10.1037// 1040-3590.1.4.331

Pettersson, I., Törnquist, K., \& Ahlström, G. (2006). The effect of an outdoor powered wheelchair on activity and participation in users with stroke. Disability \& Rehabilitation: Assistive Technology, 1, 235-243. doi:10.1080/17483100600757841

Rushton, P. W., Demers, L., \& Miller, W. C., \& CanWheel Research Team. (2012, October). Measurement properties of the Wheelchair Use Confidence Scale for Power Wheelchair Users (WheelCon-P). Paper presented at the Canadian Association on Gerontology Conference, Vancouver, BC.

Sabol, T. P., \& Haley, E. S. (2006). Wheelchair evaluation for the older adult. Clinics in Geriatric Medicine, 22, 355-375.

Sakakibara, B. M., Miller, W. C., Rushton, P. W., \& Polgar, J. M. (2018). Rasch analyses of the Wheelchair Use Confidence Scale for Power Wheelchair Users. Archives of Physical Medicine and Rehabilitation, 99, 17-25. doi:10.1016/j.apmr.2017.09.004

Salminen, A.-L., Brandt, A., Samuelsson, K., Töytäri, O., \& Malmivaara, A. (2009). Mobility devices to promote activity and participation: A systematic review. Journal of Rehabilitation Medicine, 41, 697-706. doi:10.2340/16501977-0427

Shields, M. (2004). Use of wheelchairs and other mobility support devices. Health Reports, 15(3), 37-41.

Smith, E., Kenyon, L., Field, D., \& Miller, W. (2017, February/ March). Powered wheelchair provision: Current practices and opportunities. Paper presented at the 33rd Annual International Seating Symposium, Nashville, TN.

Smith, E., Rismani, S., Mortenson, W., \& Miller, W. (in press). “A chance to try:" Exploring the clinical utility of shared control teleoperation for powered wheelchair assessment and training. American Journal of Occupational Therapy.

Thabane, L., Ma, J., Chu, R., Cheng, J., Ismaila, A., Rios, L. P., .. Goldsmith, C. H. (2010). A tutorial on pilot studies: The what, why and how. BMC Medical Research Methodology, 10, 1. doi:10. 1186/1471-2288-10 -1

Tombaugh, T. N., \& McIntyre, N. J. (1992). The Mini-Mental State Examination: A comprehensive review. Journal of the American Geriatrics Society, 40(9), 922-935.

De Vreese, L., \& Neri, M. (2001). Memory rehabilitation in Alzheimer's disease: A review of progress. International Journal of Geriatric Psychiatry, 16, 794-809.

\section{Author Biographies}

Emma M. Smith, MScOT, PhD is a trainee, International Collaboration on Repair Discoveries, Vancouver, BC, Canada. At the time of the research, she was also a doctoral student, Rehabilitation Sciences Graduate Program, University of British Columbia, Graduate Candidate, GF Strong Rehabilitation Research Program, Vancouver Coastal Health Research Institute, but has since completed her PhD.

William C. Miller, PhD, OT, is Professor, Department of Occupational Science and Occupational Therapy, University of British Columbia, Researcher, GF Strong Rehabilitation Research Program, Vancouver Coastal Health Research Institute, and Principle Investigator, International Collaboration on Repair Discoveries, Vancouver, BC, Canada.

W. Ben Mortenson, PhD, OT, is Associate Professor, Department of Occupational Science and Occupational Therapy, University of British Columbia, Investigator, GF Strong Rehabilitation Research Program, Vancouver Coastal Health Research Institute, and Principle Investigator, International Collaboration on Repair Discoveries, Vancouver, BC, Canada.

Alex Mihailidis, PhD, PEng, is Professor, Department of Occupational Science and Occupational Therapy, University of Toronto, ON, Canada. 